\title{
Martingale Solution to Stochastic Extended Korteweg-de Vries Equation
}

\author{
Anna Karczewska, Maciej Szczeciński \\ Faculty of Mathematics, Computer Science and Econometrics, University of Zielona Góra, Zielona Góra, Poland \\ Email: A.Karczewska@w mie.uz.zgora.pl,M.Szczecinski@wmie.uz.zgora.pl
}

How to cite this paper: Karczewska, A. and Szczeciński, M. (2018) Martingale Solution to Stochastic Extended Korteweg-de Vries Equation. Advances in Pure Mathematics, 8, 863-878. https://doi.org/10.4236/apm.2018.812053

Received: November 16, 2018

Accepted: December 23, 2018

Published: December 26, 2018

Copyright $\odot 2018$ by authors and Scientific Research Publishing Inc. This work is licensed under the Creative Commons Attribution International License (CC BY 4.0).

http://creativecommons.org/licenses/by/4.0/

\begin{abstract}
The deterministic extended Korteweg-de Vries equation plays an essential role in the description of the creation and propagation of nonlinear waves in many fields. We study a stochastic extended Korteweg-de Vries equation driven by a multiplicative noise in the form of a cylindrical Wiener process. We prove the existence of a martingale solution to the equation studied for all physically relevant initial conditions. The proof of the solution is based on two approximations of the problem considered and the compactness method.
\end{abstract}

\section{Keywords}

Extended Korteweg-de Vries Equation, Martingale Solution, Stochastic Fluid Dynamics

\section{(c) (i) Open Access}

\section{Introduction}

The celebrated Korteweg-de Vries equation ( $K d V$ for short) [1], derived from the set of Eulerian shallow water and long wavelength equations, becomes a paradigm in the field of nonlinear partial differential equations. KdV appears as the lowest approximations of wave motion in several fields of physics, see, e.g., monographs [2] [3] [4] [5] [6] and references therein.

$\mathrm{KdV}$ is, however, the result of an approximation of the set of the Euler equations within perturbation approach limited to the first order in expansion with respect to parameters assumed to be small. Several authors have extended $\mathrm{KdV}$ to the second order (the extended $K d V$ or $K d V 2$ ), e.g. [7]-[17], which is a more exact approximation of the Euler equations but far more difficult since it contains higher nonlinear term and higher derivatives. Despite its non-integrability, KdV2 has three forms of exact analytic solutions. There exist solitonic solutions [10], periodic cnoidal solutions [14] and periodic "superposition" solutions [15] 
[16]. These solutions have the same form as corresponding solutions to KdV but with slightly different coefficients.

A natural continuation of the study of the extended $\mathrm{KdV}$ equation seems to be considering stochastic versions of such equation. KdV2 equation driven by random noise can be a model of several kinds of waves (e.g., surface water waves, waves in plasma) influenced by random factors. Two cases of the stochastic KdV2 equation are possible: the case with additive noise and the case with the multiplicative noise. The additive case was studied by us in [18], where a mild solution to KdV2 has been established.

In this paper, we consider the stochastic extended Korteweg-de Vries equation with multiplicative random noise. We prove the existence of martingale solution to stochastic KdV2 equation driven by cylindrical Wiener process. In the proof, we generalize the methods used in papers [19] and [20]. We have to emphasize that the method used in [19] for estimations was not suitable in our case. We adapted for our purposes (proof of Lemma 2.4) the approach used by Flandoli and Gątarek in [20].

The paper is organized as follows. In Section 2, we present the initial value problem for the extended $\mathrm{KdV}$ equation. Then, we formulate the definition of the martingale solution to the problem considered. Next, we give the main result formulated in Theorem 2.1 and auxiliary results (Lemmas $2.1-2.3$ ). Section 3 contains detail proofs of Lemmas 2.2 and 2.3. In Section 4, Lemma 2.1 is proved in detail. Conclusions are contained in Section 5.

\section{Existence of Martingale Solution}

We consider initial value problem for Korteweg-de Vries type equation

$$
\left\{\begin{array}{l}
u(t, x)+\left[u_{3 x}(t, x)+u(t, x) u_{x}(t, x)+u(t, x) u_{3 x}(t, x)\right. \\
\left.\quad+3 u_{x}(t, x) u_{2 x}(t, x)\right] \mathrm{d} t=\Phi(u(t, x)) \mathrm{d} W(t), \\
u(0, x)=u_{0}(x) .
\end{array}\right.
$$

In (2.1), $W(t), t \geq 0$, is a cylindrical Wiener process, $u_{0} \in L^{2}(\mathbb{R})$ is a deterministic function, $u(\cdot, \cdot): \mathbb{R}_{+} \times \mathbb{R} \rightarrow \mathbb{R}$. Moreover, we assume that $|u(t, x)|+|u|_{L^{2}(\mathbb{R})}<\lambda<\infty, \quad \lambda>0$, for all $t \in \mathbb{R}_{+}$and $x \in \mathbb{R}$, what reflects finitness of solutions to deterministic version of the Equation (2.1) (see, e.g., [10] [15] [16]). The operator $\Phi$ is a continuous mapping from $H^{2}(\mathbb{R})$ to $L_{2}^{0}\left(L^{2}(\mathbb{R})\right)$, the space of Hilbert-Schmidt operators from $L^{2}(\mathbb{R})$ to itself. The operator $\Phi$ is such that for any $u \in H^{2}(\mathbb{R})$ the following conditions hold:

$$
\underset{\kappa_{1}, \kappa_{2}>0}{\exists} \|\left.\Phi(u(x))\right|_{L_{0}^{2}\left(L^{2}(\mathbb{R})\right)} \leq \kappa_{1} \max \left\{|u(x)|_{L^{2}(\mathbb{R})}^{2},|u(x)|_{L^{2}(\mathbb{R})}\right\}+\kappa_{2} ;
$$

there exist such functions $a, b \in L^{2}(\mathbb{R})$ with compact support, that the mapping $u \mapsto(\Phi(u) a, \Phi(u) b)_{L^{2}(\mathbb{R})}$ is continuous in topology $L_{\text {loc }}^{2}(\mathbb{R})$.

Condition (2.2) will be used in some auxiliary estimates in proofs of Lemmas 2.1 - 2.3. Condition (2.3) is used in proofs of Lemma 2.1 and Theorem 2.1 of the 
existence of martingale solution to (2.4) and (2.1), respectively.

From now on, we use the notation $\langle\cdot, \cdot\rangle$ instead of $\langle\cdot, \cdot\rangle_{L^{2}(\mathbb{R})}$.

Definition 2.1 We say that the problem (2.1) has a martingale solution on the interval $[0, T], 0<T<\infty$, if there exists a stochastic basis $\left(\Omega, \mathscr{F},\left\{\mathscr{F}_{t}\right\}_{t \geq 0}, \mathbb{P},\left\{W_{t}\right\}_{t \geq 0}\right)$, where $\left\{W_{t}\right\}_{t \geq 0}$ is a cylindrical Wiener process, and there exists the process $\{u(t, x)\}_{t \geq 0}$ adapted to the filtration $\left\{\mathscr{F}_{t}\right\}_{t \geq 0}$ with trajectories belonging to the space

$$
L^{\infty}\left(0, T ; L^{2}(\mathbb{R})\right) \cap L^{2}\left(0, T ; L_{\text {loc }}^{2}(\mathbb{R})\right) \cap \mathcal{C}\left(0, T ; H_{\text {loc }}^{s}(\mathbb{R})\right), \quad s<0, \quad \mathbb{P}-\text { a.s. }
$$

such that

$$
\begin{aligned}
& \langle u(t, x), v(x)\rangle+\int_{0}^{t}\left\langle u_{3 x}(s, x)+u(s, x) u_{x}(s, x)\right. \\
& \left.+u(s, x) u_{3 x}(s, x)+3 u_{x}(s, x) u_{2 x}(s, x), v(x)\right\rangle \mathrm{d} s \\
& =\left\langle u_{0}(x), v(x)\right\rangle+\left\langle\int_{0}^{t} \Phi(u(s, x)) \mathrm{d} W(s), v(x)\right\rangle
\end{aligned}
$$

for any $t \in[0, T]$ and $v \in H_{\text {loc }}^{1}(\mathbb{R})$.

Now, we can formulate the main result of the paper.

Theorem 2.1 For all real valued functions $u_{0} \in L^{2}(\mathbb{R})$ and $0<T<\infty$ there exists a martingale solution to (2.1) with conditions (2.2) and (2.3).

Proof. Let $\varepsilon>0$. Consider

$$
\left\{\begin{array}{l}
\mathrm{d} u^{\varepsilon}(t, x)+\left[\varepsilon u_{4 x}^{\varepsilon}(t, x)+u_{3 x}^{\varepsilon}(t, x)+u^{\varepsilon}(t, x) u_{x}^{\varepsilon}(t, x)+3 u_{x}^{\varepsilon}(t, x) u_{2 x}^{\varepsilon}(t, x)\right. \\
\left.\quad+u^{\varepsilon}(t, x) u_{3 x}^{\varepsilon}(t, x)\right] \mathrm{d} t=\Phi\left(u^{\varepsilon}(t, x)\right) \mathrm{d} W(t) \\
u_{0}^{\varepsilon}(x)=u^{\varepsilon}(0, x) .
\end{array}\right.
$$

Lemma 2.1 For any $\varepsilon>0$ there exists a martingale solution to the problem (2.4) with conditions (2.2) and (2.3).

Lemma 2.2 Let $u^{\varepsilon}$ be the martingale solution to (2.4) and let condition (2.2) hold. There exists $\varepsilon_{0}>0$, such that

$$
\begin{gathered}
\exists_{C_{1}>0} \forall_{0<\varepsilon<\varepsilon_{0}} \varepsilon \mathbb{E}\left(\left|u^{\varepsilon}(t, x)\right|_{L^{2}\left(0, T ; H^{2}(\mathbb{R})\right)}^{2}\right) \leq \tilde{C}_{1}, \\
\forall_{k \in X_{k}} \exists_{C_{2}(k)>0} \forall_{0<\varepsilon<\varepsilon_{0}} \mathbb{E}\left(\left|u^{\varepsilon}(t, x)\right|_{L^{2}\left(0, T ; H^{1}(-k, k)\right)}^{2}\right) \leq \tilde{C}_{2}(k),
\end{gathered}
$$

where $X_{k}=\left\{k>0:|k| \leq \min \left\{-x_{1}, x_{2}\right\}\right\}$.

Lemma 2.3 Let condition (2.2) hold. The family of distributions $\mathscr{L}\left(u^{\varepsilon}\right)$, where $u^{\varepsilon}$ is the martingale solution to (2.4), is tight in $L^{2}\left(0, T ; L_{\text {loc }}^{2}(\mathbb{R})\right) \cap \mathcal{C}\left(0, T ; H_{\text {loc }}^{-3}(\mathbb{R})\right)$.

(Proofs of Lemmas 2.1, 2.2 and 2.3 are given in Sections 3 and Sections 4.)

Substitute in Prohorov's theorem (e.g., see Theorem 5.1 in [21]) $S:=L^{2}\left(0, T ; L_{\text {loc }}^{2}(\mathbb{R})\right) \cap \mathcal{C}\left(0, T ; H_{l o c}^{-3}(\mathbb{R})\right)$ and $\mathscr{K}:=\left\{\mathscr{L}\left(u^{\varepsilon}\right)\right\}_{\varepsilon>0}$. Since $\mathscr{Y} \subset \mathscr{P}(S)$ is tight in $S$ (see, Lemma 2.3), then it is sequentially compact, so there exists a subsequence of $\left\{\mathscr{L}\left(u^{\varepsilon}\right)\right\}_{\varepsilon>0}$ converging to some measure $\mu$ in 鸟。

Because $\left\{\mathscr{L}\left(u^{\varepsilon}\right)\right\}_{\varepsilon>0}$ is convergent, then it is also weakly convergent. 
Therefore in Skorohod's theorem (e.g., see Theorem 6.7 in [21]) one can substitute $\mu_{\varepsilon}:=\left\{\mathscr{L}\left(u^{\varepsilon}\right)\right\}_{\varepsilon>0}$ and $\mu:=\lim _{\varepsilon \rightarrow 0} \mu_{\varepsilon}$. Then there exists a space $\left(\bar{\Omega}, \bar{F},\left\{\bar{F}_{t}\right\}_{t \geq 0}, \overline{\mathbb{P}}\right)$ and random variables $\bar{u}^{\varepsilon}, \bar{u}$ with values in $L^{2}\left(0, T ; L_{\text {loc }}^{2}(\mathbb{R})\right) \cap \varnothing\left(0, T ; H_{\text {loc }}^{-3}(\mathbb{R})\right)$ such that $\bar{u}^{\varepsilon} \rightarrow \bar{u}$ in $L^{2}\left(0, T ; L_{\text {loc }}^{2}(\mathbb{R})\right)$ and in $\mathscr{C}\left(0, T ; H_{\text {loc }}^{-3}(\mathbb{R})\right)$. Moreover $\mathscr{L}\left(\bar{u}^{\varepsilon}\right)=\mathscr{L}\left(u^{\varepsilon}\right)$.

Then due to Lemma 2.2, for any $p \in \mathbb{N}$ there exist constants $\tilde{C}_{1}(p), \tilde{C}_{2}$ such that

$$
\mathbb{E}\left(\sup _{t \in[0, T]}\left|\bar{u}^{\varepsilon}(t, x)\right|_{L^{2}(\mathbb{R})}^{2 p}\right) \leq \tilde{C}_{1}(p) \text { and } \mathbb{E}\left(\left|\bar{u}^{\varepsilon}(t, x)\right|_{L^{2}\left(0, T ; H^{2}(\mathbb{R})\right)}^{2}\right) \leq \tilde{C}_{2} .
$$

Additionally,

$$
\bar{u}^{\varepsilon}(t, x) \in L^{2}\left(0, T ; H^{1}(-k, k)\right) \cap L^{\infty}\left(0, T ; L^{2}(\mathbb{R})\right), \quad \mathbb{P}-\text { a.s. }
$$

Then one can conclude that $\bar{u}^{\varepsilon} \rightarrow \bar{u}$ weakly in $L^{2}\left(\bar{\Omega}, L^{2}\left(0, T ; H^{1}(-k, k)\right)\right)$.

Let $x \in \mathbb{R}$ be fixed and denote

$$
\begin{aligned}
M^{\varepsilon}(t):= & u^{\varepsilon}(t, x)-u_{0}^{\varepsilon}(x)+\int_{0}^{t}\left[\varepsilon u^{\varepsilon}(t, x)_{4 x}(t, x)+u^{\varepsilon}(t, x) u_{x}^{\varepsilon}(t, x)\right. \\
& \left.+u_{3 x}^{\varepsilon}(t, x)+3 u_{x}^{\varepsilon}(t, x) u_{2 x}^{\varepsilon}(t, x)+u^{\varepsilon}(t, x) u_{3 x}^{\varepsilon}(t, x)\right] \mathrm{d} s, \\
\bar{M}^{\varepsilon}(t):= & \bar{u}^{\varepsilon}(t, x)-\bar{u}_{0}^{\varepsilon}(x)+\int_{0}^{t}\left[\varepsilon \bar{u}^{\varepsilon}(t, x)_{4 x}(t, x)+\bar{u}^{\varepsilon}(t, x) \bar{u}_{x}^{\varepsilon}(t, x)\right. \\
& \left.+\bar{u}_{3 x}^{\varepsilon}(t, x)+3 \bar{u}_{x}^{\varepsilon}(t, x) \bar{u}_{2 x}^{\varepsilon}(t, x)+\bar{u}^{\varepsilon}(t, x) \bar{u}_{3 x}^{\varepsilon}(t, x)\right] \mathrm{d} s .
\end{aligned}
$$

Note, that

$$
\begin{aligned}
M^{\varepsilon}(t)= & u_{0}^{\varepsilon}(x)-\int_{0}^{t}\left[\varepsilon u^{\varepsilon}(t, x)_{4 x}(t, x)+u^{\varepsilon}(t, x) u_{x}^{\varepsilon}(t, x)+u_{3 x}^{\varepsilon}(t, x)\right. \\
& \left.+3 u_{x}^{\varepsilon}(t, x) u_{2 x}^{\varepsilon}(t, x)+u^{\varepsilon}(t, x) u_{3 x}^{\varepsilon}(t, x)\right] \mathrm{d} s+\int_{0}^{t}\left(\Phi\left(u^{\varepsilon}(s, x)\right)\right) \mathrm{d} W^{\varepsilon}(s) \\
& -u_{0}^{\varepsilon}(x)+\int_{0}^{t}\left[\varepsilon u^{\varepsilon}(t, x)_{4 x}(t, x)+u^{\varepsilon}(t, x) u_{x}^{\varepsilon}(t, x)+u_{3 x}^{\varepsilon}(t, x)\right. \\
& \left.+3 u_{x}^{\varepsilon}(t, x) u_{2 x}^{\varepsilon}(t, x)+u^{\varepsilon}(t, x) u_{3 x}^{\varepsilon}(t, x)\right] \mathrm{d} s \\
= & \int_{0}^{t}\left(\Phi\left(u^{\varepsilon}(s, x)\right)\right) \mathrm{d} W(s),
\end{aligned}
$$

so, $M^{\varepsilon}(t), t \geq 0$, is a square integrable martingale with values in $L^{2}(\mathbb{R})$, adapted to filtration $\sigma\left\{u^{\varepsilon}(s, x), 0 \leq s \leq t\right\}$ with quadratic variation

$$
\left[M^{\varepsilon}\right](t)=\int_{0}^{t} \Phi\left(u^{\varepsilon}(s, x)\right)\left[\Phi\left(u^{\varepsilon}(s, x)\right)\right]^{*} \mathrm{~d} s .
$$

Substitute in the Doob inequality (e.g., see Theorem 2.2 in [22]) $M_{t}:=M^{\varepsilon}(t)$ and $p:=2 p$. Then

$$
\mathbb{E}\left[\left(\sup _{t \in[0, T]}\left|M^{\varepsilon}(t)\right|_{L^{2}(\mathbb{R})}^{p}\right)\right] \leq\left(\frac{p}{p-1}\right)^{p} \mathbb{E}\left(\left|M^{\varepsilon}(T)\right|_{L^{2}(\mathbb{R})}\right) .
$$

Assume $0 \leq s \leq t \leq T$ and let $\varphi$ be a bounded continuous function on $L^{2}\left(0, s ; L_{l o c}^{2}(\mathbb{R})\right)$ or $C\left(0, s ; H_{l o c}^{-3}(\mathbb{R})\right)$. Let $a, b \in H_{0}^{3}(-k, k), \quad k \in \mathbb{N}$, be arbitrary and fixed. Since $M^{\varepsilon}(t)$ is a martingale and $\mathscr{L}\left(\bar{u}^{\varepsilon}\right)=\mathscr{L}\left(u^{\varepsilon}\right)$, then (see [20], pp. 377-378)

$$
\begin{aligned}
& \mathbb{E}\left(\left\langle M^{\varepsilon}(t)-M^{\varepsilon}(s) ; a\right\rangle \varphi\left(u^{\varepsilon}(t, x)\right)\right)=0, \\
& \mathbb{E}\left(\left\langle\bar{M}^{\varepsilon}(t)-\bar{M}^{\varepsilon}(s) ; a\right\rangle \varphi\left(\bar{u}^{\varepsilon}(t, x)\right)\right)=0
\end{aligned}
$$


and

$$
\begin{aligned}
& \mathbb{E}\left\{\left[\left\langle M^{\varepsilon}(t) ; a\right\rangle\left\langle M^{\varepsilon}(t) ; b\right\rangle-\left\langle M^{\varepsilon}(s) ; a\right\rangle\left\langle M^{\varepsilon}(s) ; b\right\rangle\right.\right. \\
& \left.\left.-\int_{s}^{t}\left\langle\left[\Phi\left(u^{\varepsilon}(\xi, x)\right)\right]^{*} a ;\left[\Phi\left(u^{\varepsilon}(\xi, x)\right)\right]^{*} b\right\rangle \mathrm{d} \xi\right] \varphi\left(u^{\varepsilon}(t, x)\right)\right\}=0, \\
& \mathbb{E}\left\{\left[\left\langle\bar{M}^{\varepsilon}(t) ; a\right\rangle\left\langle\bar{M}^{\varepsilon}(t) ; b\right\rangle-\left\langle\bar{M}^{\varepsilon}(s) ; a\right\rangle\left\langle\bar{M}^{\varepsilon}(s) ; b\right\rangle\right.\right. \\
& \left.\left.-\int_{s}^{t}\left\langle\left[\Phi\left(\bar{u}^{\varepsilon}(\xi, x)\right)\right]^{*} a ;\left[\Phi\left(\bar{u}^{\varepsilon}(\xi, x)\right)\right]^{*} b\right\rangle \mathrm{d} \xi\right] \varphi\left(\bar{u}^{\varepsilon}(t, x)\right)\right\}=0 .
\end{aligned}
$$

Denote

$$
\begin{aligned}
\bar{M}(t):= & \bar{u}(t, x)-\bar{u}_{0}^{\varepsilon}(x)+\int_{0}^{t}\left[\bar{u}(t, x) \bar{u}_{x}(t, x)+\bar{u}_{3 x}(t, x)\right. \\
& \left.+3 \bar{u}_{x}(t, x) \bar{u}_{2 x}(t, x)+\bar{u}(t, x) \bar{u}_{3 x}(t, x)\right] \mathrm{d} s .
\end{aligned}
$$

If $\varepsilon \rightarrow 0$, then $\bar{M}^{\varepsilon}(t) \rightarrow \bar{M}(t)$ and $\bar{M}^{\varepsilon}(s) \rightarrow \bar{M}(s), \quad \overline{\mathbb{P}}$-a.s. in $H_{l o c}^{-3}(\mathbb{R})$. Moreover, since $\varphi$ is continuous, then $\varphi\left(\bar{u}^{\varepsilon}(s, x)\right) \rightarrow \varphi(\bar{u}(s, x))$, $\overline{\mathbb{P}}$-a.s. . Therfeore, if $\varepsilon \rightarrow 0$, then

$$
\mathbb{E}\left(\left\langle\bar{M}^{\varepsilon}(t)-\bar{M}^{\varepsilon}(s) ; a\right\rangle \varphi\left(\bar{u}^{\varepsilon}(t, x)\right)\right) \rightarrow \mathbb{E}(\langle\bar{M}(t)-\bar{M}(s) ; a\rangle \varphi(\bar{u}(t, x))) .
$$

Additionaly, because (by (2.3)) $\Phi$ is a continuous operator in topology $L_{l o c}^{2}(\mathbb{R})$ and $(2.7)$ holds, therefore if $\varepsilon \rightarrow 0$, then

$$
\left\langle\left(\Phi\left(\bar{u}^{\varepsilon}(s, x)\right)\right)^{*} a ;\left(\Phi\left(\bar{u}^{\varepsilon}(s, x)\right)\right)^{*} b\right\rangle \rightarrow\left\langle(\Phi(\bar{u}(s, x)))^{*} a ;(\Phi(\bar{u}(s, x)))^{*} b\right\rangle
$$

and

$$
\begin{aligned}
& \mathbb{E}\left\{\left[\left\langle\bar{M}^{\varepsilon}(t) ; a\right\rangle\left\langle\bar{M}^{\varepsilon}(t) ; b\right\rangle-\left\langle\bar{M}^{\varepsilon}(s) ; a\right\rangle\left\langle\bar{M}^{\varepsilon}(s) ; b\right\rangle\right.\right. \\
& \left.\left.-\int_{s}^{t}\left\langle\left[\Phi\left(\bar{u}^{\varepsilon}(s, \xi)\right)\right]^{*} a ;\left[\Phi\left(\bar{u}^{\varepsilon}(s, \xi)\right)\right]^{*} b\right\rangle \mathrm{d} \xi\right] \varphi\left(\bar{u}^{\varepsilon}(t, x)\right)\right\} \\
& \rightarrow \mathbb{E}\{[\langle\bar{M}(t) ; a\rangle\langle\bar{M}(t) ; b\rangle-\langle\bar{M}(s) ; a\rangle\langle\bar{M}(s) ; b\rangle \\
& \left.\left.-\int_{s}^{t}\left\langle[\Phi(\bar{u}(s, \xi))]^{*} a ;[\Phi(\bar{u}(s, \xi))]^{*} b\right\rangle \mathrm{d} \xi\right] \varphi(\bar{u}(t, x))\right\} .
\end{aligned}
$$

Then $\bar{M}(t)$ is also a square integrable martingale adapted to the filtration $\sigma\{\bar{u}(s), 0 \leq s \leq t\}$ with quadratic variation equal $\int_{0}^{t} \Phi(\bar{u}(s, x))(\Phi(\bar{u}(s, x)))^{*} \mathrm{~d} s$.

Substitute in the representation theorem (e.g., see Theorem 8.2 in [23]), $M_{t}:=\bar{M}(t),\left[M_{t}\right]:=\int_{0}^{t} \Phi(\bar{u}(s, x)) \times(\Phi(\bar{u}(s, x)))^{*} \mathrm{~d} s$ and $\Phi(s):=\Phi(\bar{u}(s, x))$.

Then there exists a process $\tilde{M}(t)=\int_{0}^{t} \Phi(\bar{u}(s, x)) \mathrm{d} W(s)$, such that $\tilde{M}(t)=\bar{M}(t), \overline{\mathbb{P}}-$ a.s. , and

$$
\begin{aligned}
& \bar{u}(t, x)-u_{0}(x)+\int_{0}^{t}\left[\bar{u}(t, x) \bar{u}_{x}(t, x)+\bar{u}_{3 x}(t, x)\right. \\
& \left.+3 \bar{u}_{x}(t, x) \bar{u}_{2 x}(t, x)+\bar{u}(t, x) \bar{u}_{3 x}(t, x)\right] \mathrm{d} s \\
& =\int_{0}^{t} \Phi(\bar{u}(s, x)) \mathrm{d} W(s) .
\end{aligned}
$$


This implies

$$
\begin{aligned}
\bar{u}(t, x)= & u_{0}(x)-\int_{0}^{t}\left[\bar{u}(t, x) \bar{u}_{x}(t, x)+\bar{u}_{3 x}(t, x)+3 \bar{u}_{x}(t, x) \bar{u}_{2 x}(t, x)\right. \\
& \left.+\bar{u}(t, x) \bar{u}_{3 x}(t, x)\right] \mathrm{d} s+\int_{0}^{t} \Phi(\bar{u}(s, x)) \mathrm{d} W(s)
\end{aligned}
$$

so $\bar{u}(t, x)$ is a solution to (2.1), what finishes the proof of Theorem 2.1.

\section{Proofs of Lemmas 2.2 and 2.3}

Proof of Lemma 2.2 Let $p: \mathbb{R} \rightarrow \mathbb{R}$, be a smooth function fulfilling conditions

1) $p$ is increasing in $\mathbb{R}$;

2) $\forall_{x \in \mathbb{R}} \quad p>\delta_{0}>0$;

3) $\forall_{n \in \mathbb{N}}\left|\frac{\partial^{n}}{\partial x^{n}} p(x)\right|<\delta_{n}$;

4) $(\lambda-2) \delta_{2} \geq \delta_{3}$.

Let $F\left(u^{\varepsilon}\right):=\int_{X} p(x)\left(u^{\varepsilon}(x)\right)^{2} \mathrm{~d} x$. Applying the Itô formula for $F\left(u^{\varepsilon}\right)$, we obtain

$$
\begin{aligned}
\mathrm{d} F\left(u^{\varepsilon}(t, x)\right) \\
=\left\langle F^{\prime}\left(u^{\varepsilon}(t, x)\right) ; \Phi\left(u^{\varepsilon}(t, x)\right)\right\rangle \mathrm{d} W(t)-\left\langle F^{\prime}\left(u^{\varepsilon}(t, x)\right) ; \varepsilon u_{4 x}^{\varepsilon}(t, x)\right. \\
\left.+u_{3 x}^{\varepsilon}(t, x)+u^{\varepsilon}(t, x) u_{x}^{\varepsilon}(t, x)+3 u_{x}^{\varepsilon}(t, x) u_{2 x}^{\varepsilon}(t, x)+u^{\varepsilon}(t, x) u_{3 x}^{\varepsilon}(t, x)\right\rangle \mathrm{d} t \\
+\frac{1}{2} \operatorname{Tr}\left\{F^{\prime \prime}\left(u^{\varepsilon}(t, x)\right) \Phi\left(u^{\varepsilon}(t, x)\right)\left[\Phi\left(u^{\varepsilon}(t, x)\right)\right]^{*}\right\} \mathrm{d} t,
\end{aligned}
$$

where

$$
\begin{aligned}
& \left\langle F^{\prime}\left(u^{\varepsilon}(t, x)\right) ; v(t, x)\right\rangle=2 \int_{X} p(x) u^{\varepsilon}(t, x) v(t, x) \mathrm{d} x \\
& \text { and } F^{\prime \prime}\left(u^{\varepsilon}(t, x)\right) v(t, x)=2 p(x) v(t, x) .
\end{aligned}
$$

We use the following estimates from ([19], p. 242). There exist $C_{1}, C_{2}, C_{3}$, such that

$$
\begin{gathered}
\int_{\mathbb{R}} p(x) u^{\varepsilon}(t, x) u_{4 x}^{\varepsilon}(t, x) \mathrm{d} x \\
\geq \frac{1}{2} \int_{\mathbb{R}} p(x)\left[u_{2 x}^{\varepsilon}(t, x)\right]^{2} \mathrm{~d} x-C_{1}\left|u^{\varepsilon}(t, x)\right|_{L^{2}(\mathbb{R})}^{2} \\
-C_{2} \int_{\mathbb{R}} p^{\prime}(x)\left[u_{x}(t, x)\right]^{2} \mathrm{~d} x \\
\int_{\mathbb{R}} p(x) u^{\varepsilon}(t, x) u_{3 x}^{\varepsilon}(t, x) \mathrm{d} x \\
\geq \frac{3}{2} \int_{\mathbb{R}} p^{\prime}(x)\left[u_{x}^{\varepsilon}(t, x)\right]^{2} \mathrm{~d} x-\frac{1}{2} \int_{\mathbb{R}} p^{\prime \prime \prime}(x)[u(t, x)]^{2} \mathrm{~d} x ; \\
\int_{\mathbb{R}} p(x)\left[u^{\varepsilon}(t, x)\right]^{2} u_{x}^{\varepsilon}(t, x) \mathrm{d} x=-\frac{1}{3} \int_{\mathbb{R}} p^{\prime}(x)\left[u^{\varepsilon}(t, x)\right]^{3} \mathrm{~d} x \\
\geq-C_{3}\left(1+\left|u^{\varepsilon}(t, x)\right|_{L^{2}(\mathbb{R})}^{6}\right)-\frac{1}{2} \int_{\mathbb{R}} p^{\prime}(x)\left[u_{x}(t, x)\right]^{2} \mathrm{~d} x .
\end{gathered}
$$

Similarly as above, one has 


$$
\begin{aligned}
& \int_{\mathbb{R}} p(x)\left[3 u_{x}^{\varepsilon}(t, x) u_{2 x}^{\varepsilon}(t, x)+u^{\varepsilon}(t, x) u_{3 x}^{\varepsilon}(t, x)\right] \\
& =\int_{\mathbb{R}} p^{\prime \prime}(x) u_{x}^{\varepsilon}(t, x)\left[u^{\varepsilon}(t, x)\right]^{2} \mathrm{~d} x+\int_{\mathbb{R}} p^{\prime}(x)\left[u_{x}^{\varepsilon}(t, x)\right]^{2} u^{\varepsilon}(t, x) \mathrm{d} x \\
& \quad+\int_{\mathbb{R}} p(x) u^{\varepsilon}(t, x) u_{x}^{\varepsilon}(t, x) u_{2 x}^{\varepsilon}(t, x) \mathrm{d} x \\
& \geq-\frac{1}{3} \int_{\mathbb{R}} p^{\prime \prime \prime}(x)\left[u^{\varepsilon}(t, x)\right]^{3} \mathrm{~d} x-\int_{\mathbb{R}} p^{\prime}(x)\left|u^{\varepsilon}(t, x)\right|\left[u_{x}^{\varepsilon}(t, x)\right]^{2} \mathrm{~d} x \\
& \quad-\int_{\mathbb{R}} p(x)\left|u^{\varepsilon}(t, x)\right| u_{x}^{\varepsilon}(t, x) u_{2 x}^{\varepsilon}(t, x) \mathrm{d} x \\
& \geq-C_{4}\left(1+\left|u^{\varepsilon}(t, x)\right|_{L^{2}(\mathbb{R})}^{6}\right)-\frac{1}{2} \int_{\mathbb{R}} p^{\prime \prime \prime}(x)\left[u_{x}(t, x)\right]^{2} \mathrm{~d} x \\
& \quad-\lambda \int_{\mathbb{R}} p^{\prime}(x)\left[u_{x}^{\varepsilon}(t, x)\right]^{2} \mathrm{~d} x-\lambda \int_{\mathbb{R}} p(x) u_{x}^{\varepsilon}(t, x) u_{2 x}^{\varepsilon}(t, x) \mathrm{d} x \\
& =-C_{4}\left(1+\left|u^{\varepsilon}(t, x)\right|_{L^{2}(\mathbb{R})}^{6}\right)-\frac{1}{2} \int_{\mathbb{R}} p^{\prime \prime \prime}(x)\left[u_{x}(t, x)\right]^{2} \mathrm{~d} x \\
& \quad-\lambda \int_{\mathbb{R}} p^{\prime}(x)\left[u_{x}^{\varepsilon}(t, x)\right]^{2} \mathrm{~d} x+\frac{1}{2} \lambda \int_{\mathbb{R}} p^{\prime}(x)\left(u_{x}^{\varepsilon}(t, x)\right)^{2} \mathrm{~d} x .
\end{aligned}
$$

In consequence, we have

$$
\begin{aligned}
\langle & F^{\prime}\left(u^{\varepsilon}(t, x)\right) ; \varepsilon u_{4 x}^{\varepsilon}(t, x)+u_{3 x}^{\varepsilon}(t, x)+u^{\varepsilon}(t, x) u_{x}^{\varepsilon}(t, x) \\
+ & \left.3 u_{x}^{\varepsilon}(t, x) u_{2 x}^{\varepsilon}(t, x)+u^{\varepsilon}(t, x) u_{3 x}^{\varepsilon}(t, x)\right\rangle \\
\geq & \varepsilon \int_{\mathbb{R}} p(x)\left[u_{2 x}^{\varepsilon}(t, x)\right]^{2} \mathrm{~d} x-2 \varepsilon C_{1} \int_{\mathbb{R}}\left[u^{\varepsilon}(t, x)\right]^{2} \mathrm{~d} x-2 \varepsilon C_{2} \int_{\mathbb{R}} p^{\prime}(x)\left[u_{x}(t, x)\right]^{2} \mathrm{~d} x \\
& +3 \int_{\mathbb{R}} p^{\prime}(x)\left[u_{x}^{\varepsilon}(t, x)\right]^{2} \mathrm{~d} x-\int_{\mathbb{R}} p^{\prime \prime \prime}(x)[u(t, x)]^{2} \mathrm{~d} x-2 C_{3}\left(1+\left|u^{\varepsilon}(t, x)\right|_{L^{2}(\mathbb{R})}^{6}\right) \\
& -\int_{\mathbb{R}} p^{\prime}(x)\left[u_{x}(t, x)\right]^{2} \mathrm{~d} x-2 C_{4}\left(1+\left|u^{\varepsilon}(t, x)\right|_{L^{2}(\mathbb{R})}^{6}\right)-\int_{\mathbb{R}} p^{\prime \prime \prime}(x)\left[u_{x}(t, x)\right]^{2} \mathrm{~d} x \\
& -2 \lambda \int_{\mathbb{R}} p^{\prime}(x)\left[u_{x}^{\varepsilon}(t, x)\right]^{2} \mathrm{~d} x+\lambda \int_{\mathbb{R}} p^{\prime}(x)\left[u_{x}^{\varepsilon}(t, x)\right]^{2} \mathrm{~d} x \\
= & \varepsilon \int_{\mathbb{R}} p(x)\left[u_{2 x}^{\varepsilon}(t, x)\right]^{2} \mathrm{~d} x+\int_{\mathbb{R}}\left[-2 \varepsilon C_{2} p^{\prime}(x)+3 p^{\prime}(x)\right. \\
& \left.-p^{\prime}(x)-p^{\prime \prime \prime}(x)-\lambda p^{\prime}(x)\right]\left[u_{x}^{\varepsilon}(t, x)\right]^{2} \mathrm{~d} x \\
& +\int_{\mathbb{R}}\left[-2 \varepsilon C_{1}-p^{\prime \prime \prime}(x)\right]\left[u^{\varepsilon}(t, x)\right]^{2} \mathrm{~d} x-C_{5}\left(1+\left|u^{\varepsilon}(t, x)\right|_{L^{2}(\mathbb{R})}^{6}\right) \\
= & \varepsilon \int_{\mathbb{R}} p(x)\left[u_{2 x}^{\varepsilon}(t, x)\right]^{2} \mathrm{~d} x+\int_{\mathbb{R}}\left[\left(-2 \varepsilon C_{2}-\lambda+2\right) p^{\prime}(x)-p^{\prime \prime \prime}(x)\right]\left[u_{x}^{\varepsilon}(t, x)\right]^{2} \mathrm{~d} x \\
& +\int_{\mathbb{R}}\left[-2 \varepsilon C_{1}-p^{\prime \prime \prime}(x)\right]\left[u^{\varepsilon}(t, x)\right]^{2} \mathrm{~d} x-C_{5}\left(1+\left|u^{\varepsilon}(t, x)\right|_{L^{2}(\mathbb{R})}^{6}\right) \\
\geq & \varepsilon \int_{\mathbb{R}} p(x)\left[u_{2 x}^{\varepsilon}(t, x)\right]^{2} \mathrm{~d} x+\int_{\mathbb{R}}\left[2 \delta_{1} \varepsilon C_{2}+\delta_{1}(\lambda-2)-\delta_{2}\right]\left[u_{x}^{\varepsilon}(t, x)\right]^{2} \mathrm{~d} x \\
& +\int_{\mathbb{R}}\left[-2 \varepsilon C_{1}-p^{\prime \prime \prime}(x)\right]\left[u^{\varepsilon}(t, x)\right]^{2} \mathrm{~d} x-C_{5}\left(1+\left|u^{\varepsilon}(t, x)\right|_{L^{2}(\mathbb{R})}^{6}\right) \\
\geq & \varepsilon \delta \int_{\mathbb{R}}\left[u_{2 x}^{\varepsilon}(t, x)\right]^{2} \mathrm{~d} x+2 \delta_{1} \varepsilon C_{2} \int_{\mathbb{R}}\left[u_{x}^{\varepsilon}(t, x)\right]^{2} \mathrm{~d} x \\
& -\left[2 \varepsilon C_{1}+\delta_{3}\right] \int_{\mathbb{R}}\left[u^{\varepsilon}(t, x)\right]^{2} \mathrm{~d} x-C_{5}\left(1+\lambda^{6}\right) . \\
& L e t\{e\}
\end{aligned}
$$

Let $\left\{e_{1}\right\}_{i \in \mathbb{N}}$ be an orthonormal basis in $L^{2}(\mathbb{R})$. Then there exists a constant $C_{4}>0$, such that

$$
\begin{aligned}
& \operatorname{Tr}\left(F^{\prime \prime}(u) \Phi(u)[\Phi(u)]^{*}\right) \\
& =2 \sum_{i \in \mathbb{N}} \int_{\mathbb{R}} p(x)\left|\Phi\left(u^{\varepsilon}(t, x)\right) e_{i}(x)\right|^{2} \mathrm{~d} x \leq C_{4}\left|\Phi\left(u^{\varepsilon}(t, x)\right)\right|_{L_{0}^{2}\left(L^{2}(X)\right)}^{2}
\end{aligned}
$$


(by (2.2)) $\leq C_{6}\left(\kappa_{1}\left|u^{\varepsilon}(t, x)\right|_{L^{2}(X)}^{2}+\kappa_{2}\right)^{2}$.

Due to (3.3) and (3.4) we have

$$
\begin{aligned}
& \mathbb{E} F\left(u^{\varepsilon}(t, x)\right) \\
& \leq F\left(u_{0}^{\varepsilon}\right)-\varepsilon \delta \mathbb{E} \int_{0}^{t} \int_{\mathbb{R}}\left[u_{2 x}^{\varepsilon}(t, x)\right]^{2} \mathrm{~d} x-2 \delta_{1} \varepsilon C_{2} \mathbb{E} \int_{0}^{t} \int_{\mathbb{R}}\left[u_{x}^{\varepsilon}(t, x)\right]^{2} \mathrm{~d} x \\
& +\left[2 \varepsilon C_{1}+\delta_{3}\right] \mathbb{E} \int_{0}^{t} \int_{\mathbb{R}}\left[u^{\varepsilon}(t, x)\right]^{2} \mathrm{~d} x+t C_{5}\left(1+\lambda^{6}\right) \\
& +C_{6} \mathbb{E} \int_{0}^{t}\left(\kappa_{1}\left|u^{\varepsilon}(t, x)\right|_{L^{2}(\mathbb{R})}^{2}+\kappa_{2}\right)^{2} \mathrm{~d} t,
\end{aligned}
$$

so,

$$
\begin{aligned}
& \mathbb{E} F\left(u^{\varepsilon}(t, x)\right)+\varepsilon \delta \mathbb{E} \int_{0}^{t} \int_{\mathbb{R}}\left[u_{2 x}^{\varepsilon}(t, x)\right]^{2} \mathrm{~d} x \mathrm{~d} t+2 \delta_{1} \varepsilon C_{2} \mathbb{E} \int_{0}^{t} \int_{\mathbb{R}}\left[u_{x}^{\varepsilon}(t, x)\right]^{2} \mathrm{~d} x \mathrm{~d} t \\
& \leq F\left(u_{0}^{\varepsilon}\right)+\left[2 \varepsilon C_{1}+\delta_{3}\right] \mathbb{E} \int_{0}^{t} \int_{\mathbb{R}}\left[u^{\varepsilon}(t, x)\right]^{2} \mathrm{~d} x \mathrm{~d} t+C_{5} t\left(1+\lambda^{6}\right) \\
& \quad+C_{6} \mathbb{E} \int_{0}^{t}\left(\kappa_{1}\left|u^{\varepsilon}(t, x)\right|_{L^{2}(\mathbb{R})}^{2}+\kappa_{2}\right)^{2} \mathrm{~d} t \\
& =F\left(u_{0}^{\varepsilon}\right)+\left[2 \varepsilon C_{1}+\delta_{3}\right] t \lambda^{2}+C_{5} t\left(1+\lambda^{6}\right)+C_{6} t\left(\kappa_{1} \lambda^{2}+\kappa_{2}\right)^{2} \\
& \leq F\left(u_{0}^{\varepsilon}\right)+\left[2 \varepsilon C_{1}+\delta_{3}\right] T \lambda^{2}+C_{5} T\left(1+\lambda^{6}\right)+C_{6} T\left(\kappa_{1} \lambda^{2}+\kappa_{2}\right)^{2} \leq \varepsilon C_{7}+C_{8} .
\end{aligned}
$$

Let $\varepsilon_{0}>0$ be fixed. Then for all $0<\varepsilon<\varepsilon_{0}$ one has

$$
\begin{aligned}
& \varepsilon \mathbb{E}\left(\left|u^{\varepsilon}(t, x)\right|_{L^{2}\left(0, T ; H^{2}(X)\right)}^{2}\right) \\
& =\varepsilon \mathbb{E} \int_{0}^{T} \int_{\mathbb{R}}\left[u^{\varepsilon}(t, x)\right]^{2} \mathrm{~d} x \mathrm{~d} t+\varepsilon \mathbb{E} \int_{0}^{T} \int_{\mathbb{R}}\left[u_{2 x}^{\varepsilon}(t, x)\right]^{2} \mathrm{~d} x \mathrm{~d} t \\
& \leq \varepsilon T \lambda^{2}+\varepsilon \mathbb{E} \int_{0}^{T} \int_{\mathbb{R}}\left[u_{2 x}^{\varepsilon}(t, x)\right]^{2} \mathrm{~d} x \mathrm{~d} t=\varepsilon T \lambda^{2}+\varepsilon \mathbb{E} \int_{0}^{T} \int_{\mathbb{R}} \frac{1}{\delta} \delta\left[u_{2 x}^{\varepsilon}(t, x)\right]^{2} \mathrm{~d} x \mathrm{~d} t \\
& \leq \varepsilon T \lambda^{2}+\frac{1}{\delta} \varepsilon \delta \mathbb{E} \int_{0}^{T} \int_{\mathbb{R}}\left[u_{2 x}^{\varepsilon}(t, x)\right]^{2} \mathrm{~d} x \leq \varepsilon T \lambda^{2}+\frac{1}{\delta} \varepsilon\left(\varepsilon C_{7}(T)+C_{8}(T)\right) \\
& \leq \varepsilon_{0} T \lambda^{2}+\frac{\varepsilon_{0}^{2} C_{7}(T)+\varepsilon_{0} C_{8}(T)}{\delta}
\end{aligned}
$$

what proves (2.5). Moreover one has

$$
\begin{aligned}
& \mathbb{E}\left(\left|u^{\varepsilon}(t, x)\right|_{L^{2}\left(0, T ; H^{1}(-k, k)\right)}^{2}\right) \\
& =\mathbb{E} \int_{0}^{T} \int_{-k}^{k}\left[u^{\varepsilon}(t, x)\right]^{2} \mathrm{~d} x \mathrm{~d} t+\mathbb{E} \int_{0}^{T} \int_{-k}^{k}\left[u_{x}^{\varepsilon}(t, x)\right]^{2} \mathrm{~d} x \mathrm{~d} t \\
& \leq \varepsilon T \lambda^{2}+\mathbb{E} \int_{0}^{T} \int_{-k}^{k}\left[u_{x}^{\varepsilon}(t, x)\right]^{2} \mathrm{~d} x \leq \varepsilon T \lambda^{2}+\mathbb{E} \int_{0}^{T} \int_{\mathbb{R}}\left[u_{x}^{\varepsilon}(t, x)\right]^{2} \mathrm{~d} x \\
& \leq \varepsilon T \lambda^{2}+\frac{1}{2 \delta_{1} \varepsilon C_{2}} 2 \delta_{1} \varepsilon C_{2} \mathbb{E} \int_{0}^{T} \int_{\mathbb{R}}\left[u_{x}^{\varepsilon}(t, x)\right]^{2} \mathrm{~d} x \\
& \leq \varepsilon T \lambda^{2}+\frac{1}{2 \delta_{1} \varepsilon C_{2}}\left(\varepsilon C_{7}(T)+C_{8}(T)\right) \leq \varepsilon_{0} T \lambda^{2}+\frac{\varepsilon_{0} C_{7}(T)+C_{8}(T)}{2 \delta_{1} \varepsilon_{0} C_{2}},
\end{aligned}
$$

what proves inequality (2.6).

Proof of Lemma 2.3. Let $k \in \mathbb{N}$ be arbitrary fixed and let $0<\varepsilon<\varepsilon_{0}$. Then 


$$
\begin{aligned}
u^{\varepsilon}(t, x)= & u_{0}^{\varepsilon}(x)-\int_{0}^{t}\left[\varepsilon u_{4 x}^{\varepsilon}(t, x)+u_{3 x}^{\varepsilon}(t, x)+u^{\varepsilon}(t, x) u_{x}^{\varepsilon}(t, x)\right. \\
& \left.+3 u_{x}^{\varepsilon}(t, x) u_{2 x}^{\varepsilon}(t, x)+u^{\varepsilon}(t, x) u_{3 x}^{\varepsilon}(t, x)\right] \mathrm{d} s \\
& +\int_{0}^{t}\left(\Phi\left(u^{\varepsilon}(s, x)\right)\right) \mathrm{d} W(s) .
\end{aligned}
$$

Denote

$$
\begin{aligned}
& J_{1}:=u_{0}^{\varepsilon}(x) ; \quad J_{2}:=-\varepsilon \int_{0}^{t} u_{4 x}^{\varepsilon}(t, x) \mathrm{d} s \\
& J_{3}:=-\int_{0}^{t} u^{\varepsilon}(s, x) u_{x}^{\varepsilon}(s, x) \mathrm{d} s ; \quad J_{4}:=-\int_{0}^{t} u_{3 x}^{\varepsilon}(t, x) \mathrm{d} s \\
& J_{5}:=-\left(3 \int_{0}^{t} u_{x}^{\varepsilon}(s, x) u_{2 x}^{\varepsilon}(t, x) \mathrm{d} s+\int_{0}^{t} u^{\varepsilon}(t, x) u_{3 x}^{\varepsilon}(t, x) \mathrm{d} s\right) \\
& J_{6}:=\int_{0}^{t}\left(\Phi\left(u^{\varepsilon}(t, x)\right)\right) \mathrm{d} W(s) .
\end{aligned}
$$

There exists a constant $C_{1}>0$, that $\mathbb{E}\left|J_{1}\right|_{W^{1,2}\left(0, T, H^{-2}(-k, k)\right)}^{2}=C_{1}$.

There exists a constant $C_{2}>0$, such that

$$
\left|-\varepsilon u_{4 x}^{\varepsilon}(t, x)\right|_{H^{-2}(-k, k)}=\varepsilon\left|u_{4 x}^{\varepsilon}(s, x)\right|_{H^{-2}(-k, k)} \leq C_{2} \varepsilon\left|u^{\varepsilon}(s, x)\right|_{H^{-2}(-k, k)} .
$$

Therefore, due to Lemma 2.2, we can write

$$
\begin{aligned}
& \mathbb{E}\left|-\varepsilon u_{4 x}^{\varepsilon}(t, x)\right|_{L^{2}\left(0, T ; H^{-2}(-k, k)\right)}^{2}=\mathbb{E} \int_{0}^{T}\left|-\varepsilon u_{4 x}^{\varepsilon}(t, x)\right|_{H^{-2}(-k, k)}^{2} \mathrm{~d} s \\
& \leq C_{2}^{2} \varepsilon^{2} \mathbb{E} \int_{0}^{T}\left|u^{\varepsilon}(s, x)\right|_{H^{2}(-k, k)}^{2} \mathrm{~d} s \leq C_{3}(k), \text { where } C_{3}(k)>0 .
\end{aligned}
$$

So, there exists a constant $C_{4}(k)>0$, such that

$$
\mathbb{E}\left|J_{2}\right|_{W^{1,2}\left(0, T, H^{-2}(-k, k)\right)}^{2} \leq C_{4}(k)
$$

Now, we use the result from ([19], p. 243). There exists a constant $C_{5}(k)>0$, that the following inequality holds

$$
\left|u^{\varepsilon}(s, x) u_{x}^{\varepsilon}(s, x)\right|_{H^{-1}(-k, k)} \leq C_{5}(k)\left|u^{\varepsilon}(s, x)\right|_{L^{2}(-k, k)}^{\frac{3}{2}}\left|u^{\varepsilon}(s, x)\right|_{H^{1}(-k, k)}^{\frac{1}{2}} .
$$

This estimate implies the existence of a constant $C_{8}(k)>0$, such that

$$
\begin{aligned}
& \left|-u^{\varepsilon}(s, x) u_{x}^{\varepsilon}(s, x)\right|_{H^{-2}(-k, k)} \\
& =\left|u^{\varepsilon}(s, x) u_{x}^{\varepsilon}(s, x)\right|_{H^{-2}(-k, k)} \leq C_{6}\left|u^{\varepsilon}(s, x) u_{x}^{\varepsilon}(s, x)\right|_{H^{-1}(-k, k)} \\
& \leq C_{7}(k)\left|u^{\varepsilon}(s, x)\right|_{L^{2}(-k, k)}^{\frac{3}{2}}\left|u^{\varepsilon}(s, x)\right|_{H^{1}(-k, k)}^{\frac{1}{2}} \\
& \leq C_{7}(k)\left|u^{\varepsilon}(s, x)\right|_{L^{2}(-k, k)}\left|u^{\varepsilon}(s, x)\right|_{L^{2}(-k, k)}^{\frac{1}{2}}\left|u^{\varepsilon}(s, x)\right|_{H^{1}(-k, k)}^{\frac{1}{2}} \\
& \leq C_{7}(k)\left[\left(2 k \lambda^{2}\right)^{\frac{1}{2}}\right]\left|u^{\varepsilon}(s, x)\right|_{H^{1}(-k, k)}^{\frac{1}{2}} \leq C_{8}(k) \lambda\left|u^{\varepsilon}(s, x)\right|_{H^{1}(-k, k)} .
\end{aligned}
$$

Due to Lemma 2.2 there exists a constant $C_{9}(k)>0$, that we can write 


$$
\begin{aligned}
& \mathbb{E}\left|-u^{\varepsilon}(s, x) u_{x}^{\varepsilon}(s, x)\right|_{L^{2}\left(0, T ; H^{-2}(-k, k)\right)}^{2} \\
& =\mathbb{E} \int_{0}^{T}\left|-u^{\varepsilon}(s, x) u_{x}^{\varepsilon}(s, x)\right|_{H^{-2}(-k, k)}^{2} \mathrm{~d} s \\
& \leq C_{8}^{2}(k) \lambda^{2} \mathbb{E} \int_{0}^{T}\left|u^{\varepsilon}(s, x)\right|_{H^{1}(-k, k)}^{2} \mathrm{~d} s \\
& =C_{8}^{2}(k) \lambda^{2} \mathbb{E}\left|u^{\varepsilon}(s, x)\right|_{L^{2}\left(0, T ; H^{1}(-k, k)\right)}^{2} \leq C_{9}(k) \lambda^{2} .
\end{aligned}
$$

Then, there exists a constant $C_{10}(k)>0$, such that

$$
\mathbb{E}\left|J_{3}\right|_{W^{1,2}\left(0, T, H^{-2}(-k, k)\right)}^{2} \leq C_{10}(k) .
$$

We have

$$
\begin{aligned}
& \left|-u_{3 x}^{\varepsilon}(t, x)\right|_{H^{-2}(-k, k)} \\
& =\left|u_{3 x}^{\varepsilon}(t, x)\right|_{H^{-2}(-k, k)} \leq C_{11}\left|u^{\varepsilon}(s, x)\right|_{H^{1}(-k, k)} \\
& \leq C_{12}\left|u^{\varepsilon}(s, x)\right|_{H^{2}(-k, k)}, \text { where } C_{12}>0 .
\end{aligned}
$$

Lemma 2.2 implies the existence of a constant $C_{13}>0$, such that

$$
\begin{aligned}
& \mathbb{E}\left|-u_{3 x}^{\varepsilon}(t, x)\right|_{L^{2}\left(0, T ; H^{-2}(-k, k)\right)}^{2} \\
& =\mathbb{E} \int_{0}^{T}\left|-u_{3 x}^{\varepsilon}(t, x)\right|_{H^{-2}(-k, k)}^{2} \mathrm{~d} s \leq C_{12}^{2} \mathbb{E} \int_{0}^{T}\left|u^{\varepsilon}(s, x)\right|_{H^{2}(-k, k)}^{2} \mathrm{~d} s \\
& =C_{12}^{2} \mathbb{E}\left|u^{\varepsilon}(s, x)\right|_{L^{2}\left(0, T ; H^{2}(-k, k)\right)}^{2} \leq C_{12}^{2} \mathbb{E}\left|u^{\varepsilon}(s, x)\right|_{L^{2}\left(0, T ; H^{2}(\mathbb{R})\right)}^{2} \leq C_{13} .
\end{aligned}
$$

So, there exists a constant $C_{14}>0$, such that $\mathbb{E}\left|J_{4}\right|_{W^{1,2}\left(0, T, H^{-2}(-k, k)\right)}^{2} \leq C_{14}$.

There exist constants $C_{15}, C_{16}(k)>0$, that

$$
\begin{aligned}
& \left|-\left(3 u_{x}^{\varepsilon}(s, x) u_{2 x}^{\varepsilon}(t, x)+u^{\varepsilon}(t, x) u_{3 x}^{\varepsilon}(t, x)\right)\right|_{H^{-2}(-k, k)} \\
& \leq C_{15}\left|u^{\varepsilon}(s, x) u_{x}^{\varepsilon}(s, x)\right|_{H^{2}(-k, k)} \leq C_{16}(k) \lambda^{2}\left|u^{\varepsilon}(s, x)\right|_{H^{1}(-k, k)} .
\end{aligned}
$$

Due to Lemma 2.2 there exists a constant $C_{17}(k)>0$, such that

$$
\begin{aligned}
& \mathbb{E}\left|-\left(3 u_{x}^{\varepsilon}(s, x) u_{2 x}^{\varepsilon}(t, x)+u^{\varepsilon}(t, x) u_{3 x}^{\varepsilon}(t, x)\right)\right|_{L^{2}\left(0, T ; H^{-3}(-k, k)\right)}^{2} \\
& =\mathbb{E} \int_{0}^{T}\left|-\left(3 u_{x}^{\varepsilon}(s, x) u_{2 x}^{\varepsilon}(t, x)+u^{\varepsilon}(t, x) u_{3 x}^{\varepsilon}(t, x)\right)\right|_{H^{-3}(-k, k)}^{2} \mathrm{~d} s \\
& \leq C_{16}^{2}(k) \lambda^{4} \mathbb{E} \int_{0}^{T}\left|u^{\varepsilon}(s, x)\right|_{H^{1}(-k, k)}^{2} \mathrm{~d} s \\
& =C_{16}^{2}(k) \lambda^{4} \mathbb{E}\left|u^{\varepsilon}(s, x)\right|_{L^{2}\left(0, T ; H^{1}(-k, k)\right)}^{2} \leq C_{17}(k) \lambda^{4} .
\end{aligned}
$$

So, there exists a constant $C_{18}(k)>0$, that $\mathbb{E}\left|J_{5}\right|_{W^{1,2}\left(0, T, H^{-3}(-k, k)\right)}^{2} \leq C_{18}(k)$.

Substitute in ([20], Lemma 2.1) $f(s):=\Phi(u(s, x)), \quad K=H=L^{2}(\mathbb{R})$. Then $\mathcal{I}(f)(t)=\int_{0}^{t} \Phi(u(s, x)) \mathrm{d} W(s)$ and for all $p \geq 1$ and $\alpha<\frac{1}{2}$ there exists a constant $C_{22}(p, \alpha)>0$, such that 


$$
\begin{aligned}
& \mathbb{E}\left|\int_{0}^{t} \Phi\left(u^{m}(s, x)\right) \mathrm{d} W(s)\right|_{W^{\alpha(p), 2 p}\left(0, T ; L^{2}(\mathbb{R})\right)}^{2 p} \\
& \leq C_{22}(2 p, \alpha) \mathbb{E}\left(\int_{0}^{T}\left|\Phi\left(u^{m}(s, x)\right)\right|_{L_{2}^{0}\left(L^{2}(\mathbb{R})\right)}^{2 p} \mathrm{~d} s\right) .
\end{aligned}
$$

Then, due to condition (2.2), there exists a constant $C_{23}>0$, that

$$
\mathbb{E}\left|\int_{0}^{t} \Phi\left(u^{m}(s, x)\right) \mathrm{d} W(s)\right|_{W^{\alpha, 2 p}\left(0, T ; L^{2}(\mathbb{R})\right)}^{2 p} \leq C_{23}(p, \alpha) .
$$

Substitution in the above inequality $p:=1$ yields

$$
\begin{aligned}
\mathbb{E}\left|J_{6}\right|_{W^{\alpha, 2}\left(0, T ; L^{2}(\mathbb{R})\right)}^{2} & =\mathbb{E}\left|\int_{0}^{t} \Phi(u(s, x)) \mathrm{d} W(s)\right|_{W^{\alpha, 2}\left(0, T ; L^{2}(\mathbb{R})\right)}^{2} \\
& \leq C_{23}(2, \alpha)=C_{24}(\alpha) .
\end{aligned}
$$

Let $\beta \in\left(0, \frac{1}{2}\right)$ and $\alpha \in\left(\beta+\frac{1}{2}, \infty\right)$ be arbitrary fixed. Note, that the following inclusions hold

$$
W^{\alpha, 2}\left(0, T ; L^{2}(\mathbb{R})\right) \subset W^{\alpha, 2}\left(0, T ; H^{-2}(-k, k)\right) ;
$$

and $W^{1,2}\left(0, T, H^{-2}(-k, k)\right) \subset W^{\alpha, 2}\left(0, T, H^{-2}(-k, k)\right)$.

Then, there exists a constant $C_{25}(\alpha)>0$, such that

$$
\begin{aligned}
& \mathbb{E}\left|u^{m}(s, x)\right|_{W^{\alpha, 2}\left(0, T, H^{-2}(-k, k)\right)}^{2} \leq \mathbb{E}\left(\sum_{i=1}^{6}\left|J_{i}\right|_{W^{\alpha, 2}\left(0, T, H^{-2}(-k, k)\right)}\right)^{2} \\
& =\mathbb{E}\left|\sum_{i=1}^{6} J_{i}\right|_{W^{\alpha, 2}\left(0, T, H^{-2}(-k, k)\right)}^{2} \\
& =\mathbb{E}\left[\sum_{i=1}^{6}\left|J_{i}\right|_{W^{\alpha, 2}\left(0, T, H^{-2}(-k, k)\right)}^{2}+2 \sum_{i=1}^{6} \sum_{j=i+1}^{6}\left|J_{i}\right|_{W^{\alpha, 2}\left(0, T, H^{-2}(-k, k)\right)}\left|J_{j}\right|_{W^{\alpha, 2}\left(0, T, H^{-2}(-k, k)\right)}\right] \\
& \leq \mathbb{E}\left[\sum_{i=1}^{6}\left|J_{i}\right|_{W^{\alpha, 2}\left(0, T, H^{-2}(-k, k)\right)}^{2}+2 \sum_{i=1}^{6} \sum_{j=i+1}^{6}\left(\left|J_{i}\right|_{W^{\alpha, 2}\left(0, T, H^{-2}(-k, k)\right)}^{2}+\left|J_{j}\right|_{W^{\alpha, 2}\left(0, T, H^{-2}(-k, k)\right)}^{2}\right)\right] \\
& =\mathbb{E}\left[8 \sum_{i=1}^{6}\left|J_{i}\right|_{W^{\alpha, 2}\left(0, T, H^{-2}(-k, k)\right)}^{2}\right]=8 \sum_{i=1}^{6}\left[\mathbb{E}\left|J_{i}\right|_{W^{\alpha, 2}\left(0, T, H^{-2}(-k, k)\right)}^{2}\right] \leq C_{25}(\alpha) .
\end{aligned}
$$

Moreover

$$
W^{\alpha, 2}\left(0, T, H^{-2}(-k, k)\right) \subset C^{\beta}\left(0, T ; H_{l o c}^{-3}(-k, k)\right) ;
$$

and $W^{\alpha, 2}\left(0, T, H^{-2}(\mathbb{R})\right) \subset W^{\alpha, 2}\left(0, T, H^{-2}(-k, k)\right)$.

So, there exist constants $C_{27}(k), C_{28}(k, \alpha)>0$, that

$$
\begin{aligned}
& \mathbb{E}\left|u^{\varepsilon}(s, x)\right|_{C^{\beta}\left(0, T ; H^{-3}(-k, k)\right)}^{2} \leq C_{26} \mathbb{E}\left|u^{\varepsilon}(s, x)\right|_{W^{\alpha, 2}\left(0, T, H^{-3}(-k, k)\right)}^{2} \leq C_{27}(k, \alpha) \\
& \mathbb{E}\left|u^{\varepsilon}(s, x)\right|_{W^{\alpha, 2}\left(0, T, H^{-2}(-k, k)\right)} \leq C_{28}(k, \alpha) .
\end{aligned}
$$

Let $\eta>0$ be arbitrary fixed. Due to Lemma 2.2 there exists a constant $C_{30}(k)>0$, that

$$
\mathbb{E}\left|u^{\varepsilon}(s, x)\right|_{L^{2}\left(0, T, H^{-1}(-k, k)\right)}^{2} \leq C_{29}(k) \mathbb{E}\left|u^{\varepsilon}(s, x)\right|_{L^{2}\left(0, T, H^{-1}(\mathbb{R})\right)}^{2} \tilde{C}_{2}=C_{30}(k) .
$$


Substituting in ([19] Lemma 2.1) $\alpha_{k}:=\eta^{-1} 2^{k}\left(C_{30}(k)+C_{27}(k, \alpha)+C_{28}(k, \alpha)\right)$ and using Markov inequality ([24] p. 114) for

$$
\begin{aligned}
X:= & \left|u^{\varepsilon}(s, x)\right|_{L^{2}\left(0, T, H^{-1}(-k, k)\right)}^{2}+\left|u^{\varepsilon}(s, x)\right|_{W^{\alpha, 2}\left(0, T, H^{-2}(-k, k)\right)}^{2} \\
& +\left|u^{\varepsilon}(s, x)\right|_{C^{\beta}\left(0, T ; H_{\text {loc }}^{-3}(-k, k)\right)}^{2}
\end{aligned}
$$

and $\varepsilon:=\eta^{-1} 2^{k}\left(C_{30}(k)+C_{27}(k, \alpha)+C_{28}(k, \alpha)\right)$, we obtain

$$
\begin{aligned}
\mathbb{P}\left(u^{\varepsilon} \in A\left(\left\{\alpha_{k}\right\}\right)\right) \\
=1-\mathbb{P}\left(\left|u^{\varepsilon}(s, x)\right|_{L^{2}\left(0, T, H^{-1}(-k, k)\right)}^{2}+\left|u^{\varepsilon}(s, x)\right|_{W^{\alpha, 2}\left(0, T, H^{-2}(-k, k)\right)}^{2}\right. \\
\left.+\left|u^{\varepsilon}(s, x)\right|_{C^{\beta}\left(0, T ; H_{l o c}^{-3}(-k, k)\right)}^{2} \geq \eta^{-1} 2^{k}\left(C_{30}(k)+C_{27}(k, \alpha)+C_{28}(k, \alpha)\right)\right) \\
=1-\frac{C_{30}(k)+C_{27}(k, \alpha)+C_{28}(k, \alpha)}{\eta^{-1} 2^{k}\left(C_{30}(k)+C_{27}(k, \alpha)+C_{28}(k, \alpha)\right)}=1-\frac{\eta}{2^{k}}>1-\eta .
\end{aligned}
$$

Let $K$ be the following mapping for $\eta>0: K(\eta)=A\left(\left\{a_{k}^{(\eta)}\right\}\right)$, where $\left\{a_{k}^{(\eta)}\right\}$ is an increasing sequence of positive numbers, which can, but does not have to, depend on $\eta$. Note, that due to ([19] Lemma 2.1), the set $K(\eta)$ is compact for all $\eta>0$. Moreover, $\mathbb{P}\{K(\eta)\}>1-\eta$, then the family $\mathcal{L}\left(u^{\varepsilon}\right)$ is tight.

\section{Proof of Lemma 2.1}

Proof. Let $\left\{e_{i}\right\}_{i \in \mathbb{N}}$ be an orthonormal basis in space $L^{2}(\mathbb{R})$. Denote by $P_{m}$, for all $m \in \mathbb{N}$, the orthogonal projection on $\operatorname{Sp}\left(e_{0}, \cdots, e_{m}\right)$. Consider finite dimensional approximation of the problem (2.4) in the space $P_{m} L^{2}(\mathbb{R})$ of the form

$$
\left\{\begin{array}{l}
\mathrm{d} u^{m, \varepsilon}(t, x)+\left[\varepsilon \theta\left(\frac{\left|u_{4 x}^{m, \varepsilon}(t, x)\right|^{2}}{m}\right) u_{4 x}^{m, \varepsilon}(t, x)+\theta\left(\frac{\left|u_{x}^{m, \varepsilon}(t, x)\right|^{2}}{m}\right) u^{m, \varepsilon}(t, x) u_{x}^{m, \varepsilon}(t, x)\right. \\
+\theta\left(\frac{\left|u_{3 x}^{m, \varepsilon}(t, x)\right|^{2}}{m}\right) u_{3 x}^{m, \varepsilon}(t, x)+3 \theta\left(\frac{\left|u_{x}^{m, \varepsilon}(t, x) u_{2 x}^{m, \varepsilon}(t, x)\right|^{2}}{m}\right) u_{2 x}^{m, \varepsilon}(t, x) \\
\left.+\theta\left(\frac{\left|u_{3 x}^{m, \varepsilon}(t, x)\right|^{2}}{m}\right) u^{m, \varepsilon}(t, x) u_{3 x}^{m, \varepsilon}(t, x)\right] \mathrm{d} t=P_{m} \Phi\left(u^{m, \varepsilon}(t, x)\right) \mathrm{d} W^{m}(t) \\
u_{0}^{m, \varepsilon}(x)=P_{m} u^{\varepsilon}(0, x),
\end{array}\right.
$$

where $\theta \in C^{\infty}(\mathbb{R})$ fulfils conditions

$$
\begin{cases}\theta(\xi)=1, & \text { when } \xi \in[0,1] \\ \theta(\xi) \in[0,1], & \text { when } \xi \in(1,2) . \\ \theta(\xi)=0, & \text { when } \xi \in[2, \infty)\end{cases}
$$

Let $m \in \mathbb{N}$ be arbitrary fixed and 


$$
\begin{aligned}
b(u(t, x)):= & \theta\left(\frac{\left|u_{x}^{m, \varepsilon}(t, x)\right|^{2}}{m}\right) u^{m, \varepsilon}(t, x) u_{x}^{m, \varepsilon}(t, x) \\
& +\theta\left(\frac{\left|u_{3 x}^{m, \varepsilon}(t, x)\right|^{2}}{m}\right) u^{m, \varepsilon}(t, x) u_{3, x}^{m, \varepsilon}(t, x) \\
& +3 \theta\left(\frac{\left|u_{x}^{m, \varepsilon}(t, x) u_{2 x}^{m, \varepsilon}(t, x)\right|^{2}}{m}\right) u_{x}^{m, \varepsilon}(t, x) u_{2 x}^{m, \varepsilon}(t, x), \\
\sigma(u(t, x)):= & \Phi\left(u^{m, \varepsilon}(t, x)\right) .
\end{aligned}
$$

Then

$$
\begin{aligned}
|b(u(t, x))|_{L^{2}(\mathbb{R})} \leq & \left|\theta\left(\frac{\left|u_{x}^{m, \varepsilon}(t, x)\right|^{2}}{m}\right) u^{m, \varepsilon}(t, x) u_{x}^{m, \varepsilon}(t, x)\right|_{L^{2}(\mathbb{R})} \\
& +\left|\theta\left(\frac{\left|u_{3 x}^{m, \varepsilon}(t, x)\right|^{2}}{m}\right) u^{m, \varepsilon}(t, x) u_{3 x}^{m, \varepsilon}(t, x)\right|_{L^{2}(\mathbb{R})} \\
& +3\left|\theta\left(\frac{\left|u_{x}^{m, \varepsilon}(t, x) u_{2 x}^{m, \varepsilon}(t, x)\right|_{L^{2}(\mathbb{R})}^{2}}{m}\right) u_{x}^{m, \varepsilon}(t, x) u_{2 x}^{m, \varepsilon}(t, x)\right|_{L^{2}(\mathbb{R})} \\
= & J_{1}+J_{2}+3 J_{3} .
\end{aligned}
$$

Note, that

$$
J_{1}= \begin{cases}0, & \text { when }\left|u_{x}^{m, \varepsilon}(t, x)\right| \geq \sqrt{2 m} \\ \lambda\left|u^{m, \varepsilon}(t, x) u_{x}^{m, \varepsilon}(t, x)\right|_{L^{2}(\mathbb{R})}, & \text { when }\left|u_{x}^{m, \varepsilon}(t, x)\right| \leq \sqrt{2 m}\end{cases}
$$

where $\lambda \in[0,1]$, therefore

$$
J_{1} \leq\left|u^{m, \varepsilon}(t, x) u_{x}^{m, \varepsilon}(t, x)\right|_{L^{2}(\mathbb{R})} \leq \sqrt{2 m}\left|u^{m, \varepsilon}(t, x)\right|_{L^{2}(\mathbb{R})} .
$$

Analogously,

$$
J_{2} \leq\left|u^{m, \varepsilon}(t, x) u_{3 x}^{m, \varepsilon}(t, x)\right|_{L^{2}(\mathbb{R})} \leq \sqrt{2 m}\left|u^{m, \varepsilon}(t, x)\right|_{L^{2}(\mathbb{R})} .
$$

\section{Moreover}

$$
J_{3}= \begin{cases}0, & \text { when }\left|u_{x}^{m, \varepsilon}(t, x) u_{2 x}^{m, \varepsilon}(t, x)\right|_{L^{2}(\mathbb{R})}^{2} \geq \sqrt{2 m} \\ \lambda\left|u_{x}^{m, \varepsilon}(t, x) u_{2 x}^{m, \varepsilon}(t, x)\right|^{2}, & \text { when }\left|u_{x}^{m, \varepsilon}(t, x) u_{2 x}^{m, \varepsilon}(t, x)\right|_{L^{2}(\mathbb{R})}^{2} \leq \sqrt{2 m}\end{cases}
$$

where $\lambda \in[0,1]$, so

$$
3 J_{3} \leq 3\left|u^{m, \varepsilon}(t, x) u_{x}^{m, \varepsilon}(t, x)\right|_{L^{2}(\mathbb{R})} \leq 3 \sqrt{2 m} .
$$

Finally,

$$
\left|b\left(u^{m, \varepsilon}(t, x)\right)\right|_{L^{2}(\mathbb{R})} \leq 2 \sqrt{2 m}\left|u^{m, \varepsilon}(t, x)\right|_{L^{2}(\mathbb{R})}+3 \sqrt{2 m} .
$$


Additionally, due to the condition (2.2), there exist constants $\kappa_{1}, \kappa_{2}>0$, such that

$$
\left\|\Phi\left(u^{m, \varepsilon}(t, x)\right)\right\|_{L_{0}^{2}\left(L^{2}(\mathbb{R})\right)} \leq \kappa_{1}\left|u^{m, \varepsilon}(t, x)\right|_{L^{2}(\mathbb{R})}+\kappa_{2}
$$

then

$$
\begin{aligned}
& \left|b\left(u^{m, \varepsilon}(t, x)\right)\right|_{L^{2}(\mathbb{R})}+\|\left.\sigma\left(u^{m, \varepsilon}(t, x)\right)\right|_{L_{0}^{2}\left(L^{2}(\mathbb{R})\right)} \\
& \leq 2 \sqrt{2 m}\left|u^{m, \varepsilon}(t, x)\right|_{L^{2}(\mathbb{R})}+3 \sqrt{2 m}+\kappa_{1}\left|u^{m, \varepsilon}(t, x)\right|_{L^{2}(\mathbb{R})}+\kappa_{2} \\
& =\left(2 \sqrt{2 m}+\kappa_{1}\right)\left|u^{m, \varepsilon}(t, x)\right|_{L^{2}(\mathbb{R})}+3 \sqrt{2 m}+\kappa_{2} \\
& \leq\left(3 \sqrt{2 m}+\max \left\{\kappa_{1}, \kappa_{2}\right\}\right)\left|u^{m, \varepsilon}(t, x)\right|_{L^{2}(\mathbb{R})}+3 \sqrt{2 m}+\max \left\{\kappa_{1}, \kappa_{2}\right\} \\
& =\left(3 \sqrt{2 m}+\max \left\{\kappa_{1}, \kappa_{2}\right\}\right)\left(\left|u^{m, \varepsilon}(t, x)\right|_{L^{2}(\mathbb{R})}+1\right) .
\end{aligned}
$$

Therefore, from ([25] Prop. 3.6 and 4.6), when $b(u(t, x))$ and $\sigma(u(t, x))$ are as above, for all $m \in \mathbb{N}$, there exists a martingale solution to (4.1). Moreover, applying the same methods as in Section 3, using (2.3), one can show that for all $m$ the following inequalities hold

$$
\begin{gathered}
\exists_{C_{1}(\varepsilon)>0} \mathbb{E}\left(\left|u^{m, \varepsilon}(t, x)\right|_{L^{2}\left(0, T ; H^{2}(\mathbb{R})\right)}^{2}\right) \leq \tilde{C}_{1}(\varepsilon), \\
\forall_{k \in X_{k}} \exists_{C_{2}(k, \varepsilon)>0} \mathbb{E}\left(\left|u^{m, \varepsilon}(t, x)\right|_{L^{2}\left(0, T ; H^{1}(-k, k)\right)}^{2}\right) \leq \tilde{C}_{2}(k, \varepsilon) ;
\end{gathered}
$$

and the family of distributions $\mathcal{L}\left(u^{m, \varepsilon}\right)$ is tight in $L^{2}\left(0, T ; L_{\text {loc }}^{2}(\mathbb{R})\right) \cap C\left(0, T ; H_{\text {loc }}^{-3}(\mathbb{R})\right)$. Then application of the same methods, as used already on pages $3-5$, leads to the proof of the existence of martingale solution to (2.4)

\section{Conclusions}

The existence of martingale solution to highly nonlinear extended KdV equations is proved for all physically relevant initial conditions. The presence of several nonlinear terms in the considered equation required the development of much more involved methods for proving the main theorem than for the case of stochastic KdV equation.

The methods developed in the current paper can be applied to study stochastic hybrid Korteweg-de Vries-Burgers equations, particularly important for nonlinear ion-acoustic waves in plasma physics.

\section{Conflicts of Interest}

The authors declare no conflicts of interest regarding the publication of this paper.

\section{References}

[1] Korteweg, D.J. and de Vries, F. (1895) On the Change of Form of Long Waves Ad- 
vancing in a Rectangular Canal, and on a New Type of Long Stationary Waves. Philosophical Magazine, 39, 422-443.

[2] Whitham, G.B. (1974) Linear and Nonlinear Waves. Wiley, New York.

[3] Newell, A.C. (1985) Solitons in Mathematics and Physics. Society for Industrial and Applied Mathematics, Philadelphia, PAS.

[4] Drazin, P.G. and Johnson, R.S. (1989) Solitons: An Introduction. Cambridge University Press, Cambridge.

[5] Remoissenet, M. (1999) Waves Called Solitons: Concepts and Experiments. Springer, New York, Berlin.

[6] Infeld, E. and Rowlands, G. (2000) Nonlinear Waves, Solitons and Chaos. 2nd Edition, Cambridge University Press, Cambridge.

[7] Marchant, T.R. and Smyth, N.F. (1990) The Extended Korteweg-de Vries Equation and the Resonant Flow of a Fluid over Topography. Journal of Fluid Mechanics, 221, 263-288.

[8] Marchant, T.R. and Smyth, N.F. (1996) Soliton Interaction for the Extended Korteweg-de Vries Equation. IMA Journal of Applied Mathematics, 56, 157-176.

[9] Burde, G.I. and Sergyeyev, A. (2013) Ordering of Two Small Parameters in the Shallow Water Wave Problem. Journal of Physics A: Mathematical and Theoretical, 46, Article ID: 075501.

[10] Karczewska, A., Rozmej, P. and Infeld, E. (2014) Shallow-Water Soliton Dynamics beyond the Korteweg-de Vries Equation. Physical Review E, 90, Article ID: 012907. https://doi.org/10.1103/PhysRevE.90.012907

[11] Karczewska, A., Rozmej, P. and Rutkowski, L. (2014) A New Nonlinear Equation in the Shallow Water Wave Problem. Physica Scripta, 89, Article ID: 054026. https://doi.org/10.1088/0031-8949/89/5/054026

[12] Karczewska, A., Rozmej, P. and Infeld, E. (2015) Energy Invariant for Shallow-Water Waves and the Korteweg-de Vries Equation: Doubts about the Invariance of Energy. Physical Review E, 92, Article ID: 053202.

https://doi.org/10.1103/PhysRevE.92.053202

[13] Karczewska, A., Rozmej, P., Infeld, E. and Rowlands, G. (2017) Adiabatic Invariants of the Extended KdV Equation. Physics Letters A, 381, 270-275. https://doi.org/10.1016/j.physleta.2016.11.035

[14] Infeld, E., Karczewska, A., Rowlands, G. and Rozmej, P. (2018) Exact Cnoidal Solutions of the Extended KdV Equation. Acta Physica Polonica A, 133, 1191-1199. https://doi.org/10.12693/APhysPolA.133.1191

[15] Rozmej, P., Karczewska, A. and Infeld, E. (2018) Superposition Solutions to the Extended KdV Equation for Water Surface Waves. Nonlinear Dynamics, 91, 1085-1093. https://doi.org/10.1007/s11071-017-3931-1

[16] Rozmej, P. and Karczewska, A. (2018) New Exact Superposition Solutions to KdV2 Equation. Advances in Mathematical Physics, 2018, Article ID: 5095482. https://doi.org/10.1155/2018/5095482

[17] Yang, J. (2001) Dynamics of Embedded Solitons in the Extended Korteweg-de Vries Equations. Studies in Applied Mathematics, 106, 337-365. https://doi.org/10.1111/1467-9590.00169

[18] Karczewska, A. and Szczeciński, M. (2018) The Existence of Mild Solution to Stochastic Extended KdV Equation.

[19] De Bouard, A. and Debussche, A. (1998) On the Stochastic Korteweg-de Vries Equ- 
ation. Journal of Functional Analysis, 154, 215-251.

https://doi.org/10.1006/jfan.1997.3184

[20] Flandoli, F. and Gatarek, D. (1995) Martingale and Stationary Solutions for Stochastic Navier-Stokes Equations. Probability Theory and Related Fields, 102, 367-391. https://doi.org/10.1007/BF01192467

[21] Billingsley, P. (1999) Convergence of Probability Measures. John Wiley \& Sons, New York. https://doi.org/10.1002/9780470316962

[22] Gawarecki, L. and Mandrekar, V. (2011) Stochastic Differential Equations in Infinite Dimensions. Springer, New York. https://doi.org/10.1007/978-3-642-16194-0

[23] Da Prato, G. and Zabczyk, J. (1992) Stochastic Equations in Infinite Dimensions. Encyclopedia of Mathematics and Its Application, Cambridge University Press, Cambridge. https://doi.org/10.1017/CBO9780511666223

[24] Papoulis, A. (1991) Probability, Random Variables, and Stochastic Processes. 3rd Edition, McGraw-Hill, New York.

[25] Karatzas, I. and Shreve, S.E. (1991) Brownian Motion and Stochastic Calculus. 2nd Edition, Springer, New York, Berlin. 